SECTION 4. Computer science, computer engineering and automation

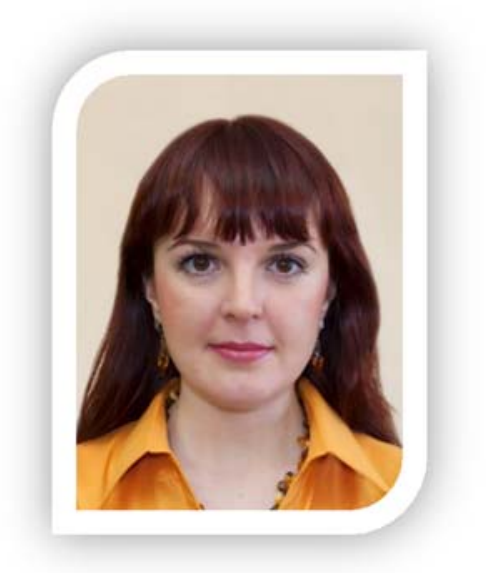

\author{
Nastashchuk Natalia Alexandrovna \\ Associate professor, Doctor of Philosophy in Pedagogy, \\ Russian Federation, Omsk, \\ Omsk State Transport University, \\ Department "Informatics, applied mathematics and \\ mechanics”
}

\title{
COMPUTATIONAL LOGIC IN ENGINEERING EDUCATION
}

The article is considered Computational Logic generally. It reveals the major and essential parts of Computational Logic applied in engineering education as well as in engineering professional activity.

Keywords: computational logic, engineering education.

In a modern informational community, an engineer professional activity is closely connected with using computers. In engineers' practice, the machine-computing technique is widely applied for computing, projecting and running of digital devices, computer-aided design (CAD), organization and planning of experimental study and research, data processing in testing of machines and mechanisms, etc. Therefore, at university engineers of all technical branches have to acquire some abilities and skills in performing industrial and scientific tasks using digital means.

One of the primary skills of engineers' informational competence is their knowledge in the field of Computational Logic (CL). It is mainly about the use of logic to perform or reason about computation. CL bears a similar relationship to computer science and engineering as mathematical logic bears to mathematics. It is synonymous with "logic in computer science" [4].

CL is a wide interdisciplinary area having its theoretical and practical roots in artificial intelligence (AA), computer science, logic, and applied mathematics. Its wideness of scope anchors in the power and generality of logic based reasoning across the spectrum of scientific disciplines, and in its practical use in the form of computer supported automated tools. CL centers around the famous definition: Algorithm = Logic + Control. According to this view, algorithms consist of a problem description (the logic part) along with a strategy to carry out useful computations on this description (the control part). The field of CL covers all kinds of applications of logic in computer science $[6,7,12]$.

CL builds upon traditional logic, which was originally developed to help people think more effectively. It employs the techniques of Symbolic Logic which is often divided into 2 branches: Propositional Logic and Predicate Logic. Symbolic Logic has been used to build the foundations of mathematics and computing [7, p.9]. The field of CL includes all types of logic (Boolean Logic, Description Logic, First-order Logic (or Predicate Calculus), Formal Logic, 
Fuzzy Logic, Mathematical Logic, Modal Logic, Predicate Logic, Propositional Logic). Boolean Logic, Propositional Logic and Predicate Calculus are the most important ones in engineers' education.

Especially in the $21^{\text {st }}$ century, the role of CL is the most important one. The $21^{\text {st }}$ century is called the age of AA. The particular feature of up-to-day machine consciousness is its ability to processing and perception of models of material real objects in the form of mathematical and logic abstractions. The fundamental base of AA is Propositional Logic, Mathematical Logic and syllogistics [Kowalski R., Lyapunov A.A., Luger G., Pospelov A.A., Pospelov D.A. et. al]. So as you can see the importance and necessity of engineers' training in the area of CL are essential.

Enhancement of technical objects, devices and systems and the development of new technological processes are possible only on the base of the most up-to-date tools and methods of data handling, methods of info-logical and mathematical modelling, methods of engineering and designing which are based on up-to-date applications of CL. So, the engineer activity has a very inventive character. That is synthesis of theory and practice, "deduction" and "production" [8]. On the one hand, an engineer has to discover original and innovative solutions in actual practice. On the other hand, he has to take into account the hard conditions of the techno-economic reality. Moreover, his invention has to bring in an economic efficiency.

There is the teaching devoted to finding some generic rules that would explain creation of new, inventive, patentable ideas [Altshuller G.S.]. It is called Theory of Inventive Problem Solving (TIPS), but it is better known by its Russian acronym TRIZ (Teoriya Resheniya Izobretatelskih Zadach). In brief, successful making decision of scientific-technical problems is implemented by means of using the methods of revealing and finding out the solutions of contradictions [Altshuller G.S., Petrov V., Shapiro R.B., Zusman A.V. et. al]. Most of these methods are based on fundamental concepts and rules of Logic, drawing conclusions, cognitive judgments and proof arguments, decision heuristics and some heuristic methods and algorithms. Therefore, CL is one of the most important and significant components of the TIPS technology.

In 1938, the Russian physicist V.I. Shestakov was the first in the world (K. Shannon did it 2 years afterwards) who proved the possibility of describing and transformation of the relay switching circuits by the methods of Boolean Logic. From that moment Practical Logic arose; it made mainly engineering tasks. Therefore, Practical Logic is also called Engineering Logic $[1,2,3,5]$. It unveils and solves graphic and analytical synthesis of combinational logical circuits (multiple-argument methods of minimization of logical functions), synthesis of microprogrammed automata on the base of integrated and relay circuits [Caldwell S.H., Lobanov V.I., Nepeivoda N.N. et. al].

Analysis and synthesis of combinational logical circuits are realized using the apparatus of Boolean Logic. It deals with logical functions which can take on a value from the set $\{0,1\}$. All these functions can be described by means of truth tables. There are 16 logical functions (or operations) for 2 arguments. The basic operations of Boolean Logic are the following ones: AND (conjunction), OR (disjunction), NOT (negation or inversion).

In Boolean Logic all operations are executed with logical variables and conformed to the laws of Boolean Logic. Actually in engineer practice are used only 2-3 laws of Boolean Logic - De Morgan's Laws and Elimination Law in the form of Karnaugh map (also known as the K-map). The K-map allows to solve a problem of minimization of logical functions finely and easily. It reduces the need for extensive calculations by taking advantage of humans' pattern-recognition capability. The algorithm of work with the K-map was worked out by V.I. Lobanov and stated in [9] more than 40 years ago.

Digital Logic is the application of Boolean Logic of 0 and 1 to electronic hardware consisting of logic gates connected to form a circuit diagram. These logical elements ANDNOT and OR-NOT are the functional base and with the help of these ones any arbitrary complex 
computer or digital electronic gate circuits can be designed. Simulation of electrical circuit is based on Boolean Logic. Automation of circuit stage in digital devices' designing is executed by using CAD, for instance, MicroSim Design Lab, PLIS, MAX+PLUS II, etc.

Now some words about another important engineer inventions having logic base. Programmable logic controllers (PLC) are widely used in the field of automation of technological processes, controlling of vehicular traffic, in communication systems, robotics technology. PLC is a controller with a programmable logic; it is an electronic component of industrial controller (a special computerized device).

Automata-based programming is a programming paradigm in which the program or its part is thought of as a model of a finite state machine or any other formal automaton. There are used such programming languages as Java, JavaSript, $\mathrm{C} / \mathrm{C}++$ and international standard IEC1131-3 (standardizes programming languages for industrial automation of PLC) [10, 11]. Also there is an instrumental tool for visual designing of automata-based program, for instance, Microsoft Domain-Specific Language Tools.

As you can see, application of CL in engineering education is major and essential: designing of computing engine on the base of synthesis of micro-programmed automaton, construction of digital systems of data processing, controlling system of technological processes, controlling system of vehicular traffic, industrial software etc.

Indeed, a good engineer is a person who is able to focus on a large variety of information and assimilate new perspective trends. But he should not to know everything. Although, is it possible to know everything about his own profession? So, at a technical university a future engineer has to study and learn the following fundamentals in the field of CL : Boolean Logic, the K-map for 8-12 variables as more effective way in handy manual minimization of logical functions; the formal synthesis of micro-programmed automaton; heuristic methods of synthesis of combinational logical circuits; basic principles of designing PLC used in the controlling systems of technological processes.

\section{References:}

1. Baranov S.I. Synthesis of micro-programmed automaton. - L.: Energy. - 1974.

2. Boiko V.I., Gurzii A.N., Zhuikov V.Y. and others. Circuit engineering of electronic system. Digital devices. - St. P.: BHV- St. Petersburg, 2004.

3. Caldwell S. H. Switching circuits and logical design. New York: John Wiley. London: Chapman and hall. 1958.

4. Computational Logic. [Electronic resource]: URL:

http://en.wikipedia.org/wiki/Computational logic (Date of access: 30.11.2013)

5. Gutnikov V.S. Integrated electronics in measuring instruments. - L.: Energy. - 1974.

6. Hölldobler S. Logik und Logikprogrammierung, Band 1: Grundlagen Synchron Publishers GmbH, 2009.

7. Kowalski R. Computational Logic and Human Thinking: How to be Artificially Intelligent. - Cambridge: Cambridge univ. press, 2011. 
8. Latipov H., Gavrilov D. and others. Engineering heuristics. [Electronic resource]: URL: http://profilib.com/chtenie/137917/nurali-latypov-inzhenernaya-evristika.php (Date of access: 30.11 .2013 )

9. Lobanov V.I. Engineering methods of implementation of digital devices. - M.: NIIRTA, 1977.

10. Shalyto A.A. Automat designing of programs. Algorithmization and programming of tasks of logic controlling // Proceedings of the Russian Academy of Sciences. Theory and controlling systems. 2000. Number 6. November-December. P. 63-81.

11. Shalyto A.A. Paradigm of automata-based programming // Scientific-technical herald StPSU ITMO. Automata-based programming. 2008. Volume 53. P. 3-23.

12. What is Computational Logic. [Electronic resource]: URL:

http://www.computational-logic.eu/intro.php (Date of access: 30.11.2013) 